\title{
Advances in infectious diseases
}

Chris Ellis

\section{Infectious diseases as a specialty}

The practice of infectious diseases (ID) is little understood by colleagues in organ-based specialties that I will start by outlining its scope. We deal with patients with every degree of immune competence with infections affecting any organ, caused by organisms ranging from those that pose no threat to other patients or carers to occasional pathogens so virulent that even experienced carers are at significant risk of fatal infection. Infectious disease specialists are daily reminded of the continuity of the living world, are aware, for example, that travel not only brings us individuals with unfamiliar diseases, unfamiliar precisely because they are not transmitted here, but can also introduce pathogens originating abroad which have the potential to flourish in a population which has no immunity to them. Our awareness of the continuity of life extends to our principal form of treatment - antibiotics - generally regarded as just one more class of pharmaceutical but, in reality, a class that is unique in participating in the most rapid process of natural selection within the living world - the biological warfare between microorganism and antimicrobial. As I review the setbacks and advances of my career lifetime I am not sure that we are even ahead on points.

\section{The animal-human link}

The statement attributed to Surgeon General William H Stewart in 1969 that 'it is time to close the book on infectious diseases' may be apocryphal but there can be no doubt that many then would have believed it. While the potential of micro-organisms to evolve resistance was already clear, few would have predicted the possibility of the postantibiotic era that we are on the brink of today. Even fewer would have foreseen the consequences of the 'animal-human link', shorthand for pathogens, particularly viruses, which pass from animals to man and which may acquire the capacity then to pass from person to person, a potential that was seen as virtually insignificant in the 'traditional' zoonoses that were mentioned in passing when I was a medical student. Related viruses co-infecting the same host may recombine to produce pathogens with transformed capacities to infect and cause disease while the speed of replication and mutation of viruses gives them the capacity to develop resistance to treatment orders of magnitude more quickly than happens in bacteria. My lifetime has seen not only the emergence of HIV infection from a simian reservoir but the virtually immediate emergence of resistance to antiretroviral drugs. The animal-human link underlies the current pandemic of dengue viral infection,

Chris Ellis, Consultant Physician, Heartlands Hospital, Birmingham spread in mosquitoes conveyed around the world in shipping containers, having probably originated in monkeys in West Africa or Eastern Asia. The risk of intruding into untamed ecosystems was well demonstrated by outbreaks of 'haemorrhagic fever with renal syndrome' in soldiers in many conflicts, especially those characterised by prolonged trench warfare. Clearly identified for the first time in soldiers fighting in Korea, the syndrome is caused by viruses, named Hantaviruses after the Hantan River in South Korea, with reservoirs in various rodents and subsequently identified throughout Eurasia and in North America. Person-to-person spread of viruses present in blood and bodily fluids pose little real threat to prepared professionals but severe acute respiratory syndrome (SARS) struck fear into the most phlegmatic. Arising out of the Chinese liking for diverse and wild sources of meat, a coronavirus, related to the cause of the common cold, causing undetectable illness in its original host, the palm civet (an animal related to the mongoose), proved to be a highly infectious, airborne pathogen. The current panzootic of influenza in domestic fowl has, thus far, only caused sporadic infection in people exposed to high doses of virus but recombination of related influenza viruses coinfecting pigs could result in hybrid viruses with both lethality and infectivity to give rise to the imminently expected avian flu pandemic.

\section{Antibiotics}

Antibiotics are without competitors as the class of pharmaceuticals with the greatest capacity to cure disease and save lives. That we regularly use them so badly is in part a reflection of our failure to deploy expertise in their use in the front line of patient care. Indeed, in most countries antibiotics are available to anyone who can afford them and it is not surprising that resistance to antituberculous therapy is rampant in areas where there is nothing to stop people using single agents if no other is available or can be afforded. But even in countries where antibiotics must be prescribed, those expert in their antimicrobial activity rarely have direct experience of their effects in patients and virtually the entire educated population, medical and lay, subscribes to the myth that 'it is vital to complete the course of an antibiotic', whereas, if we are to minimise the emergence of transmissible resistance, we should stop patients taking them as soon as they are better, except in the case of a few hard-to-treat pathogens or sites, or in immune-compromised patients. ${ }^{1}$ Only recently has there been recognition in the UK that expertise in clinical infectious diseases and in microbiology should be found in the same individual. I hope that physicians dually accredited in ID and microbiology will prove better at using and conserving antibiotics than their predecessors now that the penalty 
for their over use is not only the selection of resistance, usually a distant effect rather than one with a direct impact on the patient receiving treatment, but the emergence of one of the most unpleasant common adverse drug effects yet, Clostridium difficile infection, which ruins the lives of more people even than it kills. $C$ difficile could be a blessing in disguise if it proves to be the catalyst to physicians seriously considering the risk/benefit of antibiotic therapy, especially in the elderly who are so susceptible to the infection and from whom eradication is often impossible.

\section{Sepsis}

Bringing understanding of infection closer to patients might also lead to better management of sepsis, (ie infections with a significant systemic response) the mortality rate from which remains similar to that reported when I started my career. It is a common killer in medical and surgical wards, accounting for half of all early-warning triggers (based on points for physiological derangements calculated by nurses when they make their regular observations of vital signs). ${ }^{2}$ We understand better how infection, particularly in the blood, leads to cellular damage and disturbed physiology (though how these processes evolved remains a mystery) but, as Jonathan Cohen points out, prompt recognition followed by simple first aid, in the form of immediate parenteral antibiotics, and circulatory support, in the form of fluid challenge, would save many lives (as many as the death toll from cancer of the breast and bowel combined), requires no new technology and is now the subject of an international campaign to promote recognition and immediate treatment.

\section{Vaccination}

Vaccination has continued to make childhood safer and the universal measles, mumps, and rubella (MMR) vaccine has also reduced the incidence of congenital abnormalities. Of the vaccines we possess, we probably do not make enough use of pneumococcal vaccine in the elderly and it might also be considered as part of the cocktail of vaccines given to soldiers in expeditionary forces in whom pneumococcal infection is a significant problem, particularly at the start of overseas deployment. As hepatitis B rates rise exponentially, almost all the increase being in new immigrants, we will sooner or later need to add hepatitis $B$ vaccine to our universal schedules, given the near impossibility of comprehensively targeting the high-risk population. A wish list would include a vaccine that would protect against $C$ difficile infection but this might be easier to ask for than to deliver, given the decline in immune responsiveness with advancing age and the limited role of humoral immunity in protecting against mucosal/luminal infections. The complexity of immunity to malaria is much better understood than when I started, when I confidently expected a vaccine against Plasmodium falciparum within a decade. Now I wonder if a malaria vaccine is feasible but I hope to be proved wrong. The difficulties resulting from antigenic alteration in this parasite foreshadowed problems in developing a vaccine against HIV, a problem that I suspect will be insuperable. BCG is not protective in people in developing countries where it is most needed and an effective replacement would give some hope of reversing the malign synergy between the epidemics of HIV and tuberculosis.

\section{In conclusion}

After looking back over a career lifetime punctuated by as many setbacks as advances, what augurs well for the future and where are we not learning the lessons of the past? Campaigns raising awareness of sepsis, publicising the harm done to patients by misuse of antibiotics and the emergence of a new breed of infection specialist combining laboratory and clinical expertise will do much to offset opposing trends, which include threats to continuity of clinical care and overdefensive medical practice. Regarding the threat to human health from species-leaping pathogens I am less optimistic. Despite the power of the internet to give us instant communication, interested parties still inhabit largely separate worlds. We need better global surveillance of potentially epidemic infection in wild and domestic animals while veterinarians, public health doctors, epidemiologists and clinicians need to understand better how the others work and share their concerns and insights. I see no good reason why these barriers should not be broken down and it is just possible that we risk apocalypse by failing to do so.

\section{References}

1 Lambert HP. Don't keep taking the tablets? Lancet 1999;354:943-5.

2 Napier F, Ellis C. Sepsis accounts for half of early warning score triggers. Clin Med 2008;8:352.

Address for correspondence: Dr C Ellis, Department of Infection and Tropical Medicine, Heartlands Hospital, Birmingham B9 5SS.

Email: christopher.ellis@heartofengland.nhs.uk 